\title{
A SAÚDE DO HOMEM NA VISÃO DOS ENFERMEIROS DE UMA UNIDADE BÁSICA DE SAÚDE
}

\author{
The man's health under the nurses perspective from a basic health unit \\ La salud del hombre en la visión de los enfermeros de una unidad básica de salud \\ Patricia Alves dos Santos Silva ${ }^{1}$ \\ Monique de Sousa Furtado² \\ Aline Borges Guilhon ${ }^{3}$ \\ Norma Valéria Dantas de Oliveira Souza ${ }^{4}$ \\ Helena Maria Scherlowski Leal David 5
}

\section{RESUMO}

Este estudo objetivou conhecer e analisar a visão dos enfermeiros em relação ao atendimento à saúde do homem. Pesquisa qualitativa que teve como campo um Centro Municipal de Saúde. Os sujeitos foram sete enfermeiros que atuavam em setores que atendiam homens, dos quais três eram do sexo masculino e quatro, do sexo feminino. Utilizaram-se a entrevista com questões semiestruturadas como instrumento de coleta e a análise de conteúdo, para tratar os dados. Na fala dos sujeitos ficou evidenciado que os homens procuram menos os serviços devido à incompatibilidade de horário com a jornada laboral. $\mathrm{E}$, ainda, segundo a percepção dos sujeitos, sentem-se constrangidos em procurar atendimento, pois essa postura choca-se com a cultura andocrêntrica. Verificou-se que a maioria dos sujeitos desconhecia a Política Nacional de Atenção Integral à Saúde do Homem. Sugere-se que haja capacitação dos enfermeiros e que os serviços tenham infraestrutura física e de pessoal para garantir assistência qualificada.

Palavras-chave: Saúde do homem. Enfermagem em saúde pública. Atenção primária à saúde.

\begin{abstract}
This study was focused in understand and analyze the nurses' perspective concerning the care to the man's health. It was a qualitative research using a Municipal Health Center as a camp. Seven nurses at specific sectors for men treatment, where three were male and four female, were used as subjects. An interview with semi-structured questions was applied as a tool for data collection and content analysis, to process it. During the speech of the subjects it was evidenced that due to the incompatibility on the work schedule, men seek fewer for the services. And also, according to the perception of the subjects, they feel embarrassed to search for the treatment, as this attitude collides against the androcentric. It was noticed that most of the subjects were not aware of the National Policy for Integral Attention to Men's Health. The proposal is provide training to the nurses and that the services have physical infrastructure and staff to ensure a qualified care.
\end{abstract}

Keywords: Men's Health. Public Health Nursing. Primary Health Care.

\section{Resumen}

Este estudio tuvo como objetivo comprender y analizar la visión de los enfermeros en relación al tratamiento de la salud del hombre. Investigación cualitativa llevada a cabo en un Centro Municipal de Salud. Los sujetos fueron siete enfermeros que trabajaban en sectores que atendían a los hombres, tres de los profesionales eran hombres y cuatro, mujeres. Se utilizó la entrevista con preguntas seme-estructuradas como instrumento para la recolección y análisis de contenido para procesar los datos. En el discurso de los sujetos se evidenció que los hombres buscan menos a los servicios debido a la incompatibilidad de horario con la jornada laboral. Y, sin embargo, como es percibido por el sujeto, sienten vergüenza de buscar tratamiento, ya que este planteamiento choca con la cultura androcéntrica. Se encontró que la mayoría de los sujetos no eran conscientes de la Política Nacional de Atención Integral a la Salud del Hombre. Se sugiere que se imparta una capacitación de los enfermeros y que los servicios tengan una infraestructura física y de personal para garantizar una atención de calidad.

Palabras clave: Salud del hombre. Enfermería de Salud Pública. Atención Primaria de Salud.

\footnotetext{
'Enfermeira da Estratégia Saúde da Família da Policlínica Piquet Carneiro (PPC/UERJ). Pós-graduanda em Estomaterapia da Faculdade de Enfermagem da Universidade do Estado do Rio de Janeiro (FENF/UERJ). Rio de Janeiro-RJ. Brasil. Email: papatyenf@gmail.com; ²Enfermeira Residente em Terapia Intensiva do Hospital Universitário Pedro Ernesto.Rio de Janeiro. Rio de Janerio-RJ. Brasil. Email: blossomumu@hotmail.com; ${ }^{3}$ Enfermeira Residente em Centro Cirúrgico do Hospital Universitário Pedro Ernesto.Rio de Janeiro/RJ.Pós-graduanda em Neonatologia da Faculdade de Enfermagem da Universidade do Estado do Rio de Janeiro (FENF/UERJ). Rio de Janerio-RJ. Brasil. Email: alineguilhon@yahoo.com.br; ${ }^{4}$ Professora Adjunta do Departamento de Enfermagem Médico-cirúrgica da Faculdade de Enfermagem da Universidade do Estado do Rio de Janeiro (FENF/UERJ). Professora Permanente do Programa de Pós-graduação Stricto sensu da FENF/UERJ. Coordenadora de Ensino de Graduação da ENF/UERJ; Coordenadora do Curso em Enfermagem em Estomaterapia da ENF/UERJ. Rio de Janerio-RJ. Brasil. E-mail: norval_souza@yahoo.com.br; ${ }^{5}$ Coordenadora Adjunta de PósGraduação Scricto sensu da ENF/UERJ.Professora Adjunta do Departamento de Saúde Pública na ENF/UERJ.Rio de Janeiro-RJ. Brasil. Email: elenalealdavid@gmail.com
} 


\section{INTRODUÇÃO}

A forma como o sistema de saúde no Brasil vem se organizando revela que a maior parte do atendimento de atenção básica privilegia grupos populacionais considerados mais vulneráveis, por meio de ações programáticas voltadas para a saúde da mulher, da criança e do idoso, pouco favorecendo a atenção à saúde do homem. Pode-se constatar essa incongruência quando se analisam os programas voltados para prevenção e campanhas de autocuidado, as quais são direcionadas somente para as categorias de usuários supracitados.

Um fator que se vincula a esta problemática é a consideração de que há dificuldade, neste grupo, em reconhecer suas próprias necessidades em saúde, cultivando o pensamento que rejeita a possibilidade de adoecer, mantendo até hoje a questão cultural da invulnerabilidade masculina, de seu papel social de provedor e de herói' . Alia-se a isso a conformação do acesso aos serviços de atenção básica, historicamente estruturados para atender mulheres e crianças, e cujos horários de funcionamento coincidem com as jornadas laborais dos trabalhadores. Esta situação dificulta o atendimento de pessoas do sexo masculino, culturalmente os provedores da família e a referência como trabalhadores ${ }^{2}$.

Deve-se considerar, ainda, que, mesmo quando esses homens comparecem aos serviços de atenção básica, não significa que eles tenham suas necessidades de saúde atendidas, já que a lógica das ações programáticas não tem buscado historicamente contemplá-las, pois muitas vezes elas permanecem na lógica curativa e/ou de reabilitação?

Para minimizar tais fragilidades do sistema de saúde, o Ministério da Saúde (MS) criou a Política Nacional de Atenção Integral à Saúde do Homem - PNAISH, cujos objetivos principais são: qualificar a assistência à saúde masculina na perspectiva de linhas de cuidado que resguardem a integralidade e qualificar a atenção primária para que ela não se restrinja somente à recuperação, garantindo, sobretudo, a promoção da saúde e a prevenção de agravos evitáveis.

Cabe ressaltar que, mesmo que a criação da PNAISH seja um grande passo em direção ao aprimoramento do atendimento à saúde da população masculina, fato este que gerou um Plano de Ação Nacional com previsão de implementação entre 2009 e 2011, ainda não se tem percebido mudanças efetivas no Sistema Único de Saúde (SUS) ${ }^{3}$. Sendo assim, apesar da criação dessa política específica, os profissionais da enfermagem precisam incorporar um olhar qualificado e direcionado, que fará a assistência à saúde mais eficiente e eficaz, contribuindo para a redução de complicações e aparecimento de agravos na população masculina ${ }^{4}$.

Há, portanto, uma opção politicamente sustentada, em favor da inclusão desse grupo populacional historicamente excluído do acesso ao cuidado integral à saúde na rede pública de saúde, isto é, o desenvolvimento do cuidado em uma perspectiva interdisciplinar e multiprofissional. Isso traz, para a Enfermagem, a necessidade de rever sua prática no âmbito das equipes de saúde da atenção básica, tanto nas Estratégias Saúde da Família (ESF) como nos serviços que ainda mantêm uma organização em moldes de postos ou centros de saúde tradicionais. A atenção básica é o principal campo de empregabilidade do enfermeiro no SUS, no qual a sua atuação tem sido determinante para os avanços e consolidações obtidas $^{5}$.

Considera-se ainda importante refletir sobre as dificuldades, obstáculos e resistências associadas às especificidades do ser homem no seu processo saúde-doença, e os desafios para o seu enfrentamento pela Enfermagem na Atenção Básica. A Enfermagem tem responsabilidade nesse contexto, pois o número de pesquisas que aborda sua atuação perante a saúde do homem e, por sua vez, a baixa procura do sexo masculino pelos serviços de saúde, é uma problemática que a profissão deve ajudar a resolver ou, pelo menos, minimizar 4 .

Nesta perspectiva, considerou-se relevante desenvolver o presente estudo, que teve como objeto a visão dos enfermeiros de um serviço de atenção básica na cidade do Rio de Janeiro em relação ao atendimento à saúde do homem. E, com a finalidade de apreender 0 objeto mencionado, traçaram-se os objetivos de descrever e analisar a visão desses enfermeiros sobre 0 atendimento à saúde do homem.

Conhecer e entender o perfil masculino influenciará na conduta dos profissionais da enfermagem, visto que possibilitarão ações de saúde mais específicas e eficazes. Isso fará com que haja a adequação desses cuidados com as novas diretrizes preconizadas pelo MS. Essa evolução no atendimento acabará por promover mudanças na postura desta população, que sendo atendia com mais especialidade se sentirá menos tensa ao lidar com questões envolvendo a saúde.

\section{REVISÃO DE LITERATURA}

\section{Masculinidade e a saúde do homem}

Os estudos que vêm discutindo a saúde masculina visam a compreender as diferentes causas para os perfis de morbimortalidade e apontam como referência a perspectiva de gênero para entender o homem e seu processo saúde-doença, e os comportamentos que podem predispor a riscos de doenças e mortes ${ }^{6-7}$.

Considera-se que os modelos de masculinidade e a maneira como se dá a socialização masculina podem fragilizar ou mesmo afastar os homens das preocupações com 0 autocuidado e com a busca pelos serviços de saúde ${ }^{8}$. Dentre as questões mais frequentemente consideradas estão os valores da cultura masculina, que envolvem tendências à exposição a riscos, associação da masculinidade à invulnerabilidade e também a própria educação familiar, a qual orienta o homem para um papel social de provedor e protetor. Esta cultura tende a gerar modelos masculinos pouco aderentes a práticas de 
autocuidado e pode estimular o comportamento agressivo, violento e de descuido com ele mesmo ${ }^{9}$.

Há uma exigência, socialmente construída, de que o homem seja física e psicologicamente forte, resultando em uma figura que rejeita cuidar de si, adiando ou negando tratamentos preventivos e de promoção e de proteção da saúde. Neste sentido, verifica-se que o processo de adoecimento tornase de difícil aceitação e, embora se possa até reconhecer a importância da prevenção para a saúde em geral, não há a adoção, na prática, de tais comportamentos, nem tampouco há a busca, para fins preventivos, dos serviços de saúde, o que determina que riscos e doenças, quando existentes, sejam de difícil detecção e tratamento pelos profissionais ${ }^{9}$.

Nesta perspectiva de adoecimento masculino, podemse destacar as enfermidades da próstata, as quais configuram um grave problema de saúde pública e que têm sido alvo de atenção do MS. A alta incidência e mortalidade decorrente da neoplasia maligna de próstata fazem com que esse câncer seja o segundo mais comum em homens ${ }^{10}$. Esse tipo de doença muitas vezes se desenvolve assintomaticamente, o que, possivelmente, induz à crença de que, se os sintomas não se apresentam, o indivíduo não está doente ${ }^{8}$.

Vale destacar que um dos métodos para detecção precoce dessa neoplasia é o toque retal, mas há aspectos simbólicos que interferem na decisão do homem em realizá-lo. Esta situação afeta a sua masculinidade, criando barreiras que, em última instância, prejudicam sua saúde ${ }^{8}$.

Outro aspecto que preocupa as autoridades sanitárias em relação à saúde masculina é o hábito do tabagismo, que também é um fator de risco para doenças cardiovasculares, câncer, doenças pulmonares obstrutivas crônicas, doenças bucais e outras, relacionadas ao uso regular de cigarro.

Ainda nesse contexto, cabe lembrar sobre o câncer de pênis, que no Brasil representa $2 \%$ de todos os tipos de câncer que atingem o homem, sendo mais frequente nas regiões norte e nordeste, superando até mesmo os casos de câncer de próstata $^{11}$

Estudos científicos também sugerem a associação entre infecção pelo vírus HPV (papiloma vírus humano) e o câncer de pênis, fato que nos remete à urgência na promoção da saúde na população masculina a fim de evitar possíveis agravos associados à evolução dessa e de outras doenças ${ }^{11}$.

A influência da socialização de gênero, como também a influência das atividades laborais na construção da identidade masculina e, por sua vez, na vivência dos processos de morbimortalidade, é um fato. Neste sentido, quando se trata de cuidados com a saúde, o trabalho tem sido considerado uma barreira para o acesso aos serviços de saúde, ou a continuação de tratamentos já estabelecidos, pois há a exigência de cumprir uma jornada laboral diária e a obrigatoriedade de executar as tarefas no tempo prescrito, o que normalmente coincide com o horário de funcionamento dos serviços de saúde, quase impedindo a procura pela assistência ${ }^{6}$.
Permeando a problemática, tem-se ainda o tempo extenso para o atendimento, o longo período de espera para a efetivação deste, os intervalos prolongados entre a marcação de consulta e a realização do atendimento, e o acolhimento deficiente por parte dos profissionais ${ }^{12,13}$. Nesta perspectiva, esses também são fatores relevantes que contribuem para a situação da pouca procura da população masculina pelos serviços da saúde.

Nos aspectos estruturais, há pouco investimentos na organização do serviço em uma perspectiva de gênero, reforçando o senso comum de que os homens não são usuários da atenção primária, por isso reprimem suas necessidades de saúde e apresentam dificuldades para expressá-las, procurando menos o serviço que as mulheres ${ }^{7,12,13}$.

Além disso, a escassez de programas voltados para a saúde masculina quase inviabiliza o atendimento especializado ao homem, como, por exemplo, uma assistência sistematizada de urologia tal como acontece com as mulheres na ginecologia, em especial na atenção primária8 .

Diante dessa realidade, o MS criou a PNAISH, que atua em conjunto com a Política Nacional de Atenção Básica, com as estratégias de humanização e os princípios e diretrizes do SUS. Tal política visa a estimular o autocuidado e, sobretudo, a afirmar que a saúde é um direito social básico e de cidadania de todos os homens brasileiros ${ }^{1}$.

A PNAISH reconhece que o homem acessa o sistema de saúde por meio da atenção especializada e, para não se restringir somente à recuperação, propõe mecanismos de qualificação na atenção primária, garantindo, sobretudo, a promoção da saúde e a prevenção de agravos evitáveis' ${ }^{1}$.

A relação entre Enfermagem e a saúde do homem merece ser destacada neste trabalho, pois, durante 0 levantamento do estado da arte, pôde-se perceber a pouca produção científica - mesmo com a PNAISH - construída pelo coletivo profissional e, assim, atentar-se para importância de se ampliar esse campo de estudo.

Vale ressaltar que a categoria sofre uma persistente feminização, o que dificulta o aprofundamento das reflexões sobre o processo saúde-doença da população masculina e, por conseguinte, o não acompanhamento da evolução da sociedade por parte dos sistemas de saúde $\mathrm{e}^{7,12}$.

\section{MÉTODO}

A pesquisa teve uma abordagem qualitativa, pois, considerando a natureza do objeto de estudo, houve a necessidade de aprofundar questões relacionadas a valores, visão de mundo, crenças, percepção, entre outras de ordem subjetiva ${ }^{14}$.

0 campo de estudo foi um Centro Municipal de Saúde (CMS), localizado na zona norte da cidade do Rio de Janeiro. Esse campo foi escolhido por oferecer uma grande diversidade de serviços de saúde; dentre eles destacam-se o de cardiologia, de clínica médica e de dermatologia. Além desses serviços, 0 
CMS desenvolve uma variedade de programas de saúde, tais como: Doenças Sexualmente Transmissíveis (DST), Diabetes, Programa de Controle da Hanseníase, Hipertensão Arterial e Tratamento a Tuberculose. Desta forma, verificou-se que o campo tinha muitas possibilidades de atendimento à população masculina, mesmo que de maneira inespecífica e pouco focada nas questões de gênero, fato que proporcionou riqueza na coleta das informações.

Os sujeitos da pesquisa foram sete enfermeiros (três do sexo masculino e quatro do sexo feminino). 0 critério de inclusão para a coleta de dados era de que estes profissionais desenvolvessem suas atividades laborais junto à população masculina e ainda, que tivessem o desejo e a vontade de contribuir com a pesquisa. Sendo assim, esses enfermeiros trabalhavam nos seguintes setores: Pneumologia Sanitária, Programa de Hipertensão Arterial e Diabetes, Vacinação e Dermatologia, os quais sabidamente atendiam sistematicamente homens em suas atividades laborais. Vale informar que não havia alocação fixa e constante de enfermeiros nos outros setores do CMS, o que nos fez excluí-los como campo de estudo.

Informa-se que esta pesquisa foi aprovada pelo Comitê de Ética em Pesquisa do Município do Rio de Janeiro sob número de parecer 72/09. Enfatiza-se também que foram explicados aos sujeitos os objetivos do estudo, sua metodologia e relevância, após tal procedimento, e os enfermeiros assinaram o Termo de Consentimento Livre Esclarecido. Destaca-se, igualmente, que foi mantido o anonimato dos sujeitos, e as informações foram usadas apenas para fins científicos, os quais estão fundamentados na Resolução 196/96, que normatiza e norteia as pesquisas com seres humanos.

A coleta de dados ocorreu no período de julho a agosto do ano de 2009, por meio de entrevista com questões semiestruturadas, gravada em aparelhos de MP3, após autorização dos sujeitos. A entrevista contou com um roteiro com as seguintes perguntas: "Qual a sua visão, como enfermeiro que trabalha na atenção básica, em um Centro Municipal de Saúde de referência, em relação à saúde do homem?"; "Quais são os agravos que mais trazem o homem ao Centro Municipal de Saúde?"; "Você conhece a Política Nacional de Atenção Integral à Saúde do Homem? Fale sobre ela"; e "O que você pensa sobre a PNAISH e as medidas que podem ser tomadas para a sua implementação no Centro Municipal de Saúde?" As respostas foram posteriormente transcritas pelas próprias pesquisadoras.

Para análise das informações, foi utilizado o método de análise de conteúdo, que é um conjunto de técnicas de análise das comunicações, visando a obter, por procedimentos objetivos e sistemáticos de descrição do conteúdo das mensagens, indicadores que permitam a inferência de conhecimentos relativos às condições de produção / recepção destas mensagens ${ }^{15}$.
Optou-se, a partir da técnica de análise de conteúdo, o tipo temático, que é definido como a contagem de itens de significado ou de registro, em uma unidade de codificação determinada, e a construção de categorias. A determinação das Unidades de Significado baseou-se nas suas relações com os objetivos do estudo. Após essa etapa de codificação, agruparam-se mais uma vez as Unidades de Significação, as quais fizeram emergir 24 Unidades de Registro (UR), compondo duas categorias denominadas: Limitações para o atendimento integral à saúde do homem; Questões de gênero que implicam no atendimento à saúde do homem.

\section{RESULTADOS E DISCUSSÃO}

\section{Limitações para 0 atendimento integral à saúde do homem}

Esta categoria apresenta e discute como os enfermeiros percebem o atendimento à saúde da população masculina no local de estudo, além de analisar as dificuldades para implementação do PNAISH.

A maior parte dos discursos analisados refere-se a não existência de infraestrutura organizacional e sistematização dos serviços básicos para atender às necessidades do gênero masculino, o que foi caracterizado um sério impeditivo para um cuidado de excelência à saúde dos homens. A fala explicitada a seguir exemplifica essa análise:

O serviço de saúde também não se estrutura de forma adequada para as necessidades desse gênero. Não há infraestrutura e uma organização de trabalho que possibilite isso (E6).

As Unidades Básicas de Saúde (UBS) não disponibilizam programas ou atividades direcionadas especificamente para a população masculina, sendo esse um importante fator que indica existir uma dificuldade de interação entre as necessidades de saúde da população masculina e a organização das práticas de saúde das unidades de atenção primária”,13.

A pouca estruturação dos serviços de saúde, em termos de recursos humanos e materiais, em uma perspectiva qualitativa e quantitativa, bem como de espaço físico adequado para acolher e atender à clientela masculina, reforça a baixa procura dos homens pelos serviços de atenção primária. Não há também uma sistematização no atendimento, uma metodologia assistencial, nos poucos serviços existentes que contemplam a população em questão. Logo, a falta de diversificados recursos provoca uma queda na qualidade do atendimento, o que acaba por afastar cada vez mais o usuário ${ }^{13}$.

Um dado relevante que emergiu nos depoimentos dos sujeitos referiu-se ao pouco ou nenhum preparo dos profissionais de enfermagem na assistência à população masculina, que também recebeu destaque nas falas dos profissionais, como exemplificado a seguir: 
Eu não tenho uma visão estruturada a respeito do cuidar do homem. [...] não tenho nenhuma percepção estruturada, específica do gênero masculino. A gente ainda não criou uma base de entendimento de como construir uma ação de saúde voltada para o gênero masculino (E6).

Inferiu-se que a expressão "ausência de visão estruturada" relaciona-se a não existência de uma capacitação contínua acerca das especificidades da saúde masculina. Desse modo, sabe-se que esta é de responsabilidade da organização laboral como também do trabalhador do sistema de saúde, principalmente se considerarmos que o perfil epidemiológico da população muda e que há doenças emergentes e reemergentes. Além disso, pode-se aludir ao emprego maciço de tecnologias na área da saúde, as quais demandam aprendizagem e apropriação de seu uso com eficácia. Nesta perspectiva, aponta-se o déficit no processo de abordagem e de cuidar/cuidado da população masculina por parte dos profissionais. Igualmente, enfatizase que a ausência de acolhimento ou o acolhimento pouco atrativo está relacionado à frágil qualificação profissional para lidar com o segmento masculino ${ }^{13}$.

Vale lembrar também a importância da construção de uma postura sensível à problemática masculina desde a graduação, por meio da abordagem de conteúdos técnicocientíficos os quais fomentem uma assistência integral que atenda às complexidades relacionadas às questões de gênero, o que possibilitará desconstruir gradativamente a concepção equivocada sobre a invulnerabilidade do homem às fragilidades da vida, incluindo-se as doenças e agravos à saúde ${ }^{7,12,13}$

0 pouco número de serviços na atenção básica voltado especificamente para as demandas relacionadas às questões do gênero masculino reflete em uma organização defasada dessa infraestrutura de atendimento à saúde, e, associado à deficiência na capacitação dos profissionais que atuam nestes serviços, constitui a principal barreira para a construção de uma rede que atenda às necessidades da população masculina ${ }^{7}$. Portanto, é preciso que se volte à atenção a esta problemática a fim de favorecer o vínculo entre o homem e esses serviços.

$\mathrm{Na}$ visão dos sujeitos, revela-se grande defasagem na ofer ta de serviços de urologia na maioria das UBS, fato que influencia a procura por atendimento pelos homens. A urologia está intrinsecamente ligada à saúde masculina, pois tem como objetivo o processo terapêutico ligado à genitália masculina, além de tratar doenças do trato urinário de homens e mulheres. Uma das diretrizes da PNAISH é reorganizar as ações de saúde, por meio de uma proposta inclusiva, na qual os homens considerem os serviços de saúde também como espaços masculinos e, por sua vez, os serviços de saúde reconheçam os homens como sujeitos que necessitem de cuidados ${ }^{1}$.
São poucos os serviços que tem um serviço ambulatorial de urologia, por isso a procura do homem pelo serviço fica comprometida (E2).

Eu acho que nós, homens, somos um pouco esquecidos, eu digo isso como profissional de saúde. A saúde da mulher é muito bem focada, mas a saúde do homem não está nem se iniciando talvez porque faltam urologistas nos postos de saúde (E4).

A crítica se amplia quando se trata da inexistência de programas voltados para a saúde masculina, compartilhando a ideia de que a atenção primária não dispõe de profissionais capacitados para assistir a especificidade da saúde do homem, correlacionando situações de vida e de trabalho desta população com possíveis agravos à saúde e demarcando ações de proteção e de promoção a saúde. Fazem-se necessários um olhar direcionado aos homens, uma escuta atenta e técnica e um conhecimento epidemiológico dos problemas que os acometem ou podem acometê-los, para assim, pensar medidas para o atendimento das necessidades de saúde. Tal transformação qualitativa nos serviços de saúde ocorrerá por meio de sensibilização do coletivo profissional, do incentivo ao aprendizado, da vontade política e das desconstruções de questões de gênero que são entraves à saúde dos homens ${ }^{7,8}$.

Outro elemento encontrado na análise das Unidades de Registro foi a visão reduzida acerca da saúde do homem, pois houve tendência marcante em dimensioná-la, em especial, aos problemas da próstata. As estatísticas mostram que, comparados às mulheres, os homens utilizam mais o tabaco, usam e abusam do álcool e outras drogas, lícitas e ilícitas, apresentam uma elevada prevalência de problemas cardiovasculares, o que evidenciam outras vulnerabilidades além dos problemas de próstata ${ }^{1}$.

Todo mundo sabe que a questão da próstata tem muita divulgação, mas no centro de saúde não tem ainda, pelo menos não no nosso, um programa específico para atender ao homem na questão da próstata. Acho isto básico (E5).

Em contraponto, surgiu na fala de um dos entrevistados a necessidade de uma reorientação do atendimento, evidenciando uma visão mais aprofundada e consolidada acerca do processo saúde-doença dos homens.

A gente está enfocando mais a questão da próstata, mas compreendemos também que a saúde do homem está relacionada a outras questões, como 
combate ao tabagismo e às mudanças de estilo de vida (E2).

Ainda que se reivindique a urologia como especialidade na UBS, com a justificativa de que o generalista não dá conta de atender à especificidade do homem, faz-se necessário evitar uma visão reducionista ou fragmentalizadora da atenção primária ${ }^{8}$. Assim, vale ressaltar que a visão desses profissionais deve ser reformulada para que haja uma oferta mais ampla e qualificada, proporcionando assim um atendimento integral e, portanto, mais eficiente e eficaz.

Quando questionados sobre a PNAISH, a grande maioria dos enfermeiros afirmou desconhecê-la. Além disso, referiram como sendo relevante o fato de haver pouco conhecimento dos profissionais de saúde, principalmente os de enfermagem, e da própria população a respeito dessa Política.

Ressalvo que, por mais que seja uma ação do Ministério da Saúde, os profissionais de atenção à saúde ainda desconhecem essa Política e, então, se nós que estamos na assistência desconhecemos, ainda mais a população (E2).

Os entrevistados também fizerem uma associação entre a criação da Política e o início do processo de reavaliação da saúde do homem e de sua rede de referência e contrarreferência dentro do SUS.

Essa ação demonstra o início de todo um processo de reavaliação de reflexão acerca da saúde do homem (E6).

Essa questão vai ter que ser muito bem trabalhada e muito bem divulgada pelo Ministério da Saúde e pelas secretarias estaduais, até chegar ao ponto in loco que são as UBS, que são um referencial, realmente, de atendimento da nossa população para a promoção à saúde e prevenção (E5).

A compreensão das barreiras institucionais é importante para a proposição estratégica de medidas que venham a promover o acesso dos homens aos serviços de atenção primária, que deve ser a porta de entrada ao sistema de saúde, a fim de resguardar a promoção e a prevenção como eixos fundamentais de intervenção".

Uma estratégia apresentada pelos sujeitos da pesquisa foi a estruturação do atendimento e a criação de uma rede de referência e contrarreferência como medida que visa a resolutividade das ações no atendimento à população masculina.

Essa rede tem que estar bem estruturada, deve ser criada toda uma rede de referências e contrarreferências (E2).
A integralidade pode ser compreendida a partir da perspectiva de uma linha de cuidado estabelecendo uma dinâmica de referência e contrarreferência entre a atenção primária e a secundária e a terciária, assegurando a continuidade no processo de atenção e cuidado" ${ }^{1}$.

Compreende-se que a criação dessa política, embora retardatária, é crucial para que se inicie uma modificação na maneira de tratar e acolher a população masculina nos serviços de saúde. No entanto, sabemos da necessidade de se investir na divulgação dessa nova ação tanto para os profissionais como para a população.

\section{Questões de gênero que implicam no atendimento à saúde do homem}

Nesta categoria discutem-se as construções sociais de gênero que repercutem negativamente na saúde do homem e que limitam a procura da população masculina a um atendimento na perspectiva da proteção e da promoção da saúde.

Os homens têm dificuldades em reconhecer suas necessidades, cultivando o pensamento mágico de que não adoecem. A doença é considerada como um sinal de fragilidade que os homens não compreendem como inerente à sua própria condição sociopsíquica e biológica'.

A sociedade impõe ao homem uma postura de potência e invulnerabilidade, não the dando o direito de transparecer suas fragilidades. Não é permitido ao homem chorar, se emocionar, evidenciar o medo ou a ansiedade. Sendo assim, procurar um serviço de saúde para tratamento ou prevenção de riscos é um ato de fragilidade que se choca com as concepções desta sociedade androcêntrica?.

Embora a literatura destaque 0 aspecto sociocultural acerca do que se espera da população masculina, somente um sujeito ratificou essa prerrogativa.

O homem é ainda muito resistente a procurar um tratamento médico [...] às vezes o próprio homem culturalmente não quer parecer que está doente porque mostra fraqueza (E3).

Nesse contexto, a maioria dos homens só procura os serviços de saúde em geral quando apresenta um quadro agudo de alteração da saúde. Neste sentido, enfatiza-se essa falta de caráter preventivo quando o MS aponta para a necessidade de oferecer subsídios à reflexão dos determinantes da saúde do homem, bem como ressalta diversos elementos condicionantes para a sua saúde, destacando a relevância de ações de promoção e de prevenção, além de medidas de recuperação dirigidas à população masculina'. E esta questão aparece nas falas dos sujeitos:

A minha experiência tem me mostrado justamente isso, os homens não procuram a unidade para orientação, prevenção de alguma patologia. Quando eles buscam já estão com alguma doença instalada (E4). 
Compreendemos que é preciso incentivar o distanciamento desse modelo dito biomédico, prescritivo e não preventivo que ainda predomina nos serviços e na cultura masculina e, com isso buscar um olhar integral no atendimento. A integralidade prevê uma visão holística do ser humano, no qual se preconizam as necessidades que vão além daquilo que a clínica pode detectar.

Outro aspecto relevante que surgiu da análise foi que as UBS foram organizadas para o funcionamento em horários quase sempre incompatíveis com a jornada laboral da maioria das pessoas. Muitos homens, ao contrário da maioria das mulheres, não priorizam o cuidado à saúde em detrimento de suas tarefas. Sendo assim, procurar um atendimento de saúde muitas vezes significa, para o gênero masculino, ausentar-se do trabalho, colocando em risco sua subsistência econômica; deixa, desse modo, sua saúde em patamar de escolha secundário, o que não se verifica com a maioria das mulheres ${ }^{6}$.

Até mesmo porque os serviços de saúde da atenção básica funcionam das 7 às 17h. Então, é um horário em que muitos ainda estão cumprindo a sua jornada de trabalho (E2).

Sabemos muito bem que esse perfil da população, na sua maioria, é de trabalhadores. Então, na medida em que vocênão oferece serviço de atenção básica em horários que possam ser conciliatórios com o trabalho desses indivíduos, isto representa uma barreira de acesso (E6).

Faz-se relevante analisar e reconfigurar a organização do trabalho e o processo laboral do sistema de saúde. A sociedade vive uma produtividade ininterrupta, em que 0 consumo de bens e serviço está se tornando diuturno; neste sentido, há de se adequar a oferta de ações de saúde a fim de abranger e captar o máximo de pessoas possível. E nesta perspectiva, a organização do trabalho precisa ser flexível, ou seja, dispor de mais possibilidades de ações, e não fragmentada, procurando concentrar o máximo de cuidados em mesmos horários e lugares, a fim de favorecer uma assistência de qualidade e includente 5 .

Acima de tudo, um reposicionamento profissional deve ocorrer para a construção de um paradigma de cuidado coerente com as necessidades sentidas por cada tipo de indivíduo e, assim, melhorar a qualidade de vida e de saúde da população ${ }^{16}$.

\section{CONSIDERAÇÕES FINAIS}

Por meio deste estudo ficou evidente que, embora haja a PNAISH, é grande a necessidade de conhecer as políticas e os programas e implementar um programa de capacitação para os profissionais de saúde, principalmente os de enfermagem, para que esses possam assistir a população masculina, atendendo a suas demandas a partir de suas características.

Verificou-se a necessidade de estruturar os serviços de saúde em termos de organização e processo do trabalho a fim de atender à especificidade dessa população. Aludese à premência de adequar qualitativa e quantitativamente os recursos humanos e materiais a essa demanda específica, de oferecer fácil acesso a serviços estreitamente ligados ao homem e de readequar instalações físicas e horários de funcionamento do serviço para favorecer a assistência.

Constatou-se que é preciso desconstruir a ideia de invulnerabilidade, que impede o homem de procurar a prevenção nos serviços de saúde. É necessário permitir à população masculina a expressão de seus medos, ansiedades, fragilidades, para que se sintam mais acolhidos e possam procurar ajuda às suas questões de saúde.

Compreende-se, assim, que a criação dessa Política, embora retardatária, é crucial para que se inicie uma modificação na maneira de tratar e acolher os homens nos serviços de saúde. Porém, há necessidade de se investir na divulgação dessa ação tanto para os profissionais como para a população, através, por exemplo, dos meios de comunicação.

Este estudo buscou evidenciar a premência da readequação dos sistemas de saúde, em especial os de atenção primária, para atender às demandas do grupo masculino que ainda encontra-se mal assistido. Considerase que mais estudos como este sejam elaborados a fim de desenvolverem estratégias que venham a responder aos anseios e às necessidades dos homens. Neste sentido, sugere-se que se elaborem pesquisas cujos sujeitos sejam os homens, possibilitando que eles expressem seus anseios, suas percepções sobre questões de saúde-doença, favorecendo momentos de reflexão que possam auxiliar na transformação de uma assistência que ainda se encontra muito incipiente na oferta de ações de saúde à população masculina.

\section{REFERÊNCIAS}

1. Ministério da Saúde (BR), Departamento de Ações Programáticas Estratégicas. Política Nacional de Atenção Integral à Saúde do Homem: princípios e diretrizes. [on- line]. Braślia(DF); 2008 [citado 2008 ago 12]. Disponível em: http://www.saude.gov.br/sas/portarias.

2. Schraiber LB, Figueiredo WS, Gomes R, Couto MT, Pinheiro TF, Machin R, et al. Necessidades de saúde e masculinidades: atenção primária no cuidado aos homens. Cad Saude Publica. [on- line]. 2010 maio; [citado 2011 abr 04]; 26(5): 961-70. Disponível em: http://www.scielo.br/pdf/ csp/v26n5/18.pdf. 
3. Ministério da Saúde (BR), Departamento de Ações Programáticas Estratégicas. Política Nacional de Atenção Integral à Saúde do Homem. Plano de Ação Nacional (2009-2011). [on-line]. Brasilia(DF); 2009 [citado 2010 ago 12]; Disponível em: <http://portal.saude.gov.br/portal/ arquivos/pdf/plano_saude_homem.

4. Silva SO. Cuidado na perspectiva de homens: um olhar da enfermagem. [dissertação]. Santa Maria: Universidade Federal de Santa Maria; 2010. 97 p. [citado 04 abr. 2011]. Disponível em: http://www.ufsm.br/ppgenf/ DissertSILVANAOSILVA.pdf.

5. David HMSL, Mauro MYC, Silva VG, Pinheiro MAS, Silva FH. Organização do trabalho de enfermagem na atenção básica: uma questão para a saúde do trabalhador. Texto\&Contexto Enferm. [on-line]. 2009 [citado $2010 \mathrm{abr} 7]$; 8(2). Disponível em:http://www.scielo.br/ scielo.php?script=sdi_arttext\&pid=S010407072009000200002\&ling=pt\&nrm=iso.

6. Schraiber LB, Gomes R, Couto MT. Homens e saúde na pauta da saúde coletiva. Cienc Saude Colet. [on-line]. 2005 [citado 2008 dez 28]; 10(1):717. Disponível em: http://www.scielo.br/scielo.php.

7. Figueiredo W. Assistência à saúde dos homens: um desafio para os serviços de atenção primária. Cienc Saude Colet. [on- line]. 2005 [citado 2008 dez 28];10(1): 105-09. Disponível em: http://www.scielo.br/ scielo.php?script=sci_arttext\&pid=S1413-81232005000100017.

8. Gomes R, Nascimento EF, Rebello LEFS, Araújo FC. As arranhaduras da masculinidade: uma discussão sobre o toque retal como medida de prevenção do câncer prostático. Cienc Saude Colet. [on- line]. 2008 [citado 2008 nov 15]; 13(6): 1975-84. Disponível em: http:// www.scielo.br/scielo.php.

9. Gomes R, Nascimento EF. A produção do conhecimento da saúde pública sobre a relação homem-saúde: uma revisão bibliográfica. Cad Saude Publica. [on- line]. 2006 [citado 2009 jan 07]; 22(5): 901-11. Disponível em: http://www.ensp.fiocruz.br/csp/pes.html.

10. Ministério da Saúde (BR), Instituto Nacional do Câncer- INCA. Estimativa de incidência câncer no Brasil em 2008. [on-line]. Rio de Janeiro; 2008 [citado 2008 dez 12]. Disponível em: http://www.inca.gov.br/ conteudo_view.asp?id=1796.

11. Fonseca AG, Pinto JASA, Marques MC, Drosdoski FS, Neto LORF. Estudo epidemiológicos do câncer de pênis no estado do Pará, Brasil. Rev Pan-Amazonica Saude. [on line].2010 [citado 2010 nov 10]; 1(2): 85-90. Disponível em: http://scielo.iec.pa.gov.br/

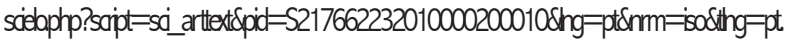

12. Gomes R, Nascimento EF, Araújo FC. Por que os homens buscam menos os serviços de saúde do que as mulheres? As explicações de homens com baixa escolaridade e homens com ensino superior. Cad Saude Publica. [on line]. 2007 [citado 2009 jan 10]; 23(3):565-74. Disponível em: http://www.scielo.br/pdf/csp/v23n3/15.pdf.

13. Beck CLC, Lisbôa RL, Tavares JP, Silva RM, Prestes FC. Humanização da assistência de enfermagem: percepção de enfermeiros nos serviços de saúde de um município. Rev Gauch Enferm [on-line]. 2009 [citado 2010 abr 12]; 30(1): 54-61. Disponível em: http://www.seer.ufrgs.br/ index.php/RevistaGauchadeEnfermagem/article/view/5102/65
14. Minayo MCS. Pesquisa social: teoria, métodos e criatividade. $28^{\mathrm{a}}$ ed. Petrópolis(RJ): Vozes; 2009.

15. Bardin L. Análise de conteúdo. Lisboa: Ed 70; 2002.

16. Oliveira DC, Costa CPM, Gomes AMT, Pontes APM. Construção de um paradigma de cuidado de enfermagem pautado nas necessidades humanas e de saúde. Esc Anna Nery. [on-line]. 2011. [citado 2012 fev 12]; 15(4):838-44. Disponível em: http://www.scielo.br/ scielo.php?pid=S1414-81452011000400025\&script=sci_arttext 